Article

\title{
The Langevin Equation in Terms of Generalized Liouville-Caputo Derivatives with Nonlocal Boundary Conditions Involving a Generalized Fractional Integral
}

\author{
Bashir Ahmad ${ }^{1}$ (D), Madeaha Alghanmi ${ }^{1}$, Ahmed Alsaedi ${ }^{1}$ and Hari M. Srivastava ${ }^{2,3, *}$ \\ and Sotiris K. Ntouyas ${ }^{1,4}$ (D) \\ 1 Nonlinear Analysis and Applied Mathematics (NAAM)-Research Group, Department of Mathematics, \\ Faculty of Science, King Abdulaziz University, P.O. Box 80203, Jeddah 21589, Saudi Arabia; \\ bashirahmad_qau@yahoo.com (B.A.); madeaha@hotmail.com (M.A.); aalsaedi@hotmail.com (A.A.); \\ sntouyas@uoi.gr (S.K.N.) \\ 2 Department of Mathematics and Statistics, University of Victoria, Victoria, BC V8W 3R4, Canada \\ 3 Department of Medical Research, China Medical University Hospital, China Medical University, \\ Taichung 40402, Taiwan \\ 4 Department of Mathematics, University of Ioannina, 45110 Ioannina, Greece \\ * Correspondence: harimsri@math.uvic.ca
}

Received: 25 April 2019; Accepted: 6 June 2019; Published: 11 June 2019

\begin{abstract}
In this paper, we establish sufficient conditions for the existence of solutions for a nonlinear Langevin equation based on Liouville-Caputo-type generalized fractional differential operators of different orders, supplemented with nonlocal boundary conditions involving a generalized integral operator. The modern techniques of functional analysis are employed to obtain the desired results. The paper concludes with illustrative examples.
\end{abstract}

Keywords: Langevin equation; generalized fractional integral; generalized Liouville-Caputo derivative; nonlocal boundary conditions; existence; fixed point

\section{Introduction}

The topic of fractional calculus has emerged as an interesting area of investigation in view of its widespread applications in social sciences, engineering and technical sciences. Mathematical models based on fractional order differential and integral operators are considered to be more realistic and practical than their integer-order counterparts as such models can reveal the history of the ongoing phenomena in systems and processes. This branch of mathematical analysis is now very developed and covers a wide range of interesting results, for instance [1-7].

The Langevin equation is an effective tool of mathematical physics, which can describe processes like anomalous diffusion in a descent manner. Examples of such processes include price index fluctuations [8], harmonic oscillators [9], etc. A generic Langevin equation for noise sources with correlations also plays a central role in the theory of critical dynamics [10]. The nature of the quantum noise can be understood better by means of a generalized Langevin equation [11]. The role of the Langevin equation in fractional systems, such as fractional reaction-diffusion systems [12,13], is very rich and beautiful. The fractional analogue (also known as the stochastic differential equation) of the usual Langevin equation is suggested for systems in which the separation between microscopic and macroscopic time scales is not observed; for example, see [8]. In [14], the author investigated moments, variances, position and velocity correlation for a Riemann-Liouville-type fractional Langevin equation 
in time and compared the results obtained with the ones derived for the same generalized Langevin equation involving the Liouville-Caputo fractional derivative. Some recent results on the Langevin equation with different boundary conditions can be found in the papers [15-20] and the references cited therein.

Motivated by the aforementioned work on the Langevin equation and its variants, in this paper, we introduce and study a new form of Langevin equation involving generalized Liouville-Caputo derivatives of different orders and solve it with nonlocal generalized fractional integral boundary conditions. In precise terms, we investigate the problem:

$$
\left\{\begin{array}{l}
{ }_{c}^{\rho} D_{a+}^{\alpha}\left({ }_{c}^{\rho} D_{a+}^{\beta}+\lambda\right) x(t)=f(t, x(t)), t \in J:=[a, T], \quad \lambda \in \mathbb{R}, \\
x(a)=0, x(\eta)=0, \quad x(T)=\mu^{\rho} I_{a+}^{\gamma} x(\xi), \quad a<\eta<\xi<T,
\end{array}\right.
$$

where ${ }_{c}^{\rho} D_{a+,}^{\alpha}{ }_{c}^{\rho} D_{a+}^{\beta}$ denote the Liouville-Caputo-type generalized fractional differential operators of order $1<\alpha \leq 2,0<\beta<1, \rho>0$, respectively, ${ }^{\rho} I_{a+}^{\gamma}$ is the generalized fractional integral operator of order $\gamma>0$ and $\rho>0$, and $f:[a, T] \times \mathbb{R} \rightarrow \mathbb{R}$ is a given continuous function.

Here, we emphasize that the present work may have useful applications in fractional quantum mechanics and fractional statistical mechanics, in relation to further generalization of the Feynman and Weiner path integrals [21].

We compose the rest of the article as follows. Section 2 contains the basic concepts of generalized fractional calculus and an auxiliary lemma dealing with the linear variant of the given problem. In Section 3, we present the main results and illustrative examples.

\section{Preliminaries}

Definition 1 ([22]). The generalized left-sided fractional integral of order $\beta>0$ and $\rho>0$ of $g \in X_{c}^{p}(a, b)$ for $-\infty<a<t<b<\infty$, is defined by:

$$
\left({ }^{\rho} I_{a+}^{\beta} g\right)(t)=\frac{\rho^{1-\beta}}{\Gamma(\beta)} \int_{a}^{t} \frac{s^{\rho-1}}{\left(t^{\rho}-s^{\rho}\right)^{1-\beta}} g(s) d s,
$$

where $X_{c}^{p}(a, b)$ denotes the space of all complex-valued Lebesgue measurable functions $\phi$ on $(a, b)$ equipped with the norm:

$$
\|\phi\|_{X_{c}^{p}}=\left(\int_{a}^{b}\left|x^{c} \phi(x)\right|^{p} \frac{d x}{x}\right)^{1 / p}<\infty, c \in \mathbb{R}, 1 \leq p \leq \infty .
$$

Similarly, the right-sided fractional integral ${ }^{\rho} I_{b-}^{\beta} g$ is defined by:

$$
\left({ }^{\rho} I_{b-}^{\beta} g\right)(t)=\frac{\rho^{1-\alpha}}{\Gamma(\beta)} \int_{t}^{b} \frac{s^{\rho-1}}{\left(s^{\rho}-t^{\rho}\right)^{1-\beta}} g(s) d s
$$

Definition 2 ([23]). For $\beta>0, n=[\beta]+1, \rho>0$ and $0 \leq a<x<b<\infty$, we define the generalized fractional derivatives in terms of the generalized fractional integrals (2) and (3) as:

$$
\begin{aligned}
\left({ }^{\rho} D_{a+}^{\beta} g\right)(t) & =\left(t^{1-\rho} \frac{d}{d t}\right)^{n}\left({ }^{\rho} I_{a+}^{n-\beta} g\right)(t) \\
& =\frac{\rho^{\beta-n+1}}{\Gamma(n-\beta)}\left(t^{1-\rho} \frac{d}{d t}\right)^{n} \int_{a}^{t} \frac{s^{\rho-1}}{\left(t^{\rho}-s^{\rho}\right)^{\beta-n+1}} g(s) d s,
\end{aligned}
$$

and:

$$
\left({ }^{\rho} D_{b-}^{\beta} g\right)(t)=\left(-t^{1-\rho} \frac{d}{d t}\right)^{n}\left({ }^{\rho} I_{b-}^{n-\beta} g\right)(t)
$$




$$
=\frac{\rho^{\beta-n+1}}{\Gamma(n-\beta)}\left(-t^{1-\rho} \frac{d}{d t}\right)^{n} \int_{t}^{b} \frac{s^{\rho-1}}{\left(s^{\rho}-t^{\rho}\right)^{\beta-n+1}} g(s) d s,
$$

if the integrals in the above expressions exist.

Definition 3 ([24]). For $\beta>0, n=[\beta]+1$ and $g \in A C_{\delta}^{n}[a, b]$, the Liouville-Caputo-type generalized fractional derivatives ${ }_{c}^{\rho} D_{a+g}^{\beta}$ and ${ }_{c}^{\rho} D_{b-g}^{\beta}$ are respectively defined via (4) and (5) as follows:

$$
\begin{gathered}
\rho_{c} D_{a+\delta}^{\beta} g(x)={ }^{\rho} D_{a+}^{\beta}\left[g(t)-\sum_{k=0}^{n-1} \frac{\delta^{k} g(a)}{k !}\left(\frac{t^{\rho}-a^{\rho}}{\rho}\right)^{k}\right](x), \delta=x^{1-\rho} \frac{d}{d x} \\
{ }_{c}^{\rho} D_{b-}^{\beta} g(x)={ }^{\rho} D_{b-}^{\beta}\left[g(t)-\sum_{k=0}^{n-1} \frac{(-1)^{k} \delta^{k} g(b)}{k !}\left(\frac{b^{\rho}-t^{\rho}}{\rho}\right)^{k}\right](x), \delta=x^{1-\rho} \frac{d}{d x}
\end{gathered}
$$

where $A C_{\delta}^{n}[a, b]$ denotes the class of all absolutely-continuous functions $g$ possessing $\delta^{n-1}$-derivative $\left(\delta^{n-1} g \in\right.$ $A C([a, b], \mathbb{R}))$, equipped with the norm $\|g\|_{A C_{\delta}^{n}}=\sum_{k=0}^{n-1}\left\|\delta^{k} g\right\|_{C}$.

Remark 1 ([24]). For $\alpha \geq 0$ and $g \in A C_{\delta}^{n}[a, b]$, the left and right generalized Liouville-Caputo derivatives of $g$ are respectively defined by the expressions:

$$
\begin{gathered}
{ }_{c}^{\rho} D_{a+}^{\beta} g(t)=\frac{1}{\Gamma(n-\beta)} \int_{a}^{t}\left(\frac{t^{\rho}-s^{\rho}}{\rho}\right)^{n-\beta-1} \frac{\left(\delta^{n} g\right)(s) d s}{s^{1-\rho}}, \\
{ }_{c}^{\rho} D_{b-}^{\beta} g(t)=\frac{1}{\Gamma(n-\beta)} \int_{t}^{b}\left(\frac{s^{\rho}-t^{\rho}}{\rho}\right)^{n-\alpha-1} \frac{(-1)^{n}\left(\delta^{n} g\right)(s) d s}{s^{1-\rho}} .
\end{gathered}
$$

Lemma 1 ([24]). Let $g \in A C_{\delta}^{n}[a, b]$ or $C_{\delta}^{n}[a, b]$ and $\beta \in \mathbb{R}$. Then:

$$
\begin{gathered}
\rho I_{a+c}^{\beta \rho} D_{a+g}^{\beta} g(x)=g(x)-\sum_{k=0}^{n-1} \frac{\left(\delta^{k} g\right)(a)}{k !}\left(\frac{x^{\rho}-a^{\rho}}{\rho}\right)^{k}, \\
\rho I_{b-c}^{\beta \rho} D_{b-}^{\beta} g(x)=g(x)-\sum_{k=0}^{n-1} \frac{(-1)^{k}\left(\delta^{k} g\right)(a)}{k !}\left(\frac{b^{\rho}-x^{\rho}}{\rho}\right)^{k} .
\end{gathered}
$$

In particular, for $0<\beta \leq 1$, we have:

$$
\rho_{I_{a+c}}^{\beta \rho} D_{a+g}^{\beta} g(x)=g(x)-g(a),{ }^{\rho} I_{b^{-}}^{\beta} \rho^{\rho} D_{b^{-}}^{\beta} g(x)=g(x)-g(b) .
$$

Definition 4. A function $x \in C([a, T], \mathbb{R})$ is called a solution of $(1)$ if $x$ satisfies the equation ${ }_{c}^{\rho} D_{a+}^{\alpha}\left({ }_{c}^{\rho} D_{a+}^{\beta}+\right.$ $\lambda) x(t)=f(t, x(t))$ on $[a, T]$, and the conditions $x(a)=0, x(\eta)=0, x(T)=\mu^{\rho} I_{a+}^{\gamma} x(\xi)$.

In the next lemma, we solve the linear variant of Problem (1).

Lemma 2. Let $h \in C([a, T], \mathbb{R}), x \in A C_{\delta}^{3}(J)$ and:

$$
\Omega=\left[\frac{\left(T^{\rho}-a^{\rho}\right)^{\beta}\left(T^{\rho}-\eta^{\rho}\right)}{\rho^{\beta+1} \Gamma(\beta+2)}-\frac{\mu\left(\xi^{\rho}-a^{\rho}\right)^{\beta+\gamma}\left[(\beta+1)\left(\xi^{\rho}-\eta^{\rho}\right)-\gamma\left(\eta^{\rho}-a^{\rho}\right)\right]}{\rho^{\beta+\gamma+1} \Gamma(\beta+\gamma+2)(\beta+1)}\right] \neq 0 .
$$

Then, the unique solution of linear problem:

$$
\left\{\begin{array}{l}
{ }_{c}^{\rho} D_{a+}^{\alpha}\left({ }_{c}^{\rho} D_{a+}^{\beta}+\lambda\right) x(t)=h(t), \quad t \in J:=[a, T], \\
x(a)=0, \quad x(\eta)=0, \quad x(T)=\mu^{\rho} I_{a+}^{\gamma} x(\xi), \quad a<\eta<\xi<T,
\end{array}\right.
$$


is given by:

$$
\begin{aligned}
x(t)= & { }^{\rho} I_{a+}^{\alpha+\beta} h(t)-\lambda^{\rho} I_{a+}^{\beta} x(t)+\frac{\left(t^{\rho}-a^{\rho}\right)^{\beta}\left(\eta^{\rho}-t^{\rho}\right)}{\rho^{\beta+1} \Gamma(\beta+2) \Omega}\left\{{ }^{\rho} I_{a+}^{\alpha+\beta} h(T)-\lambda^{\rho} I_{a+}^{\beta} x(T)\right. \\
& \left.-\mu^{\rho} I_{a+}^{\alpha+\beta+\gamma} h(\xi)+\mu \lambda^{\rho} I_{a+}^{\beta+\gamma} x(\xi)\right\}-\frac{\left(t^{\rho}-a^{\rho}\right)^{\beta}}{\Omega\left(\eta^{\rho}-a^{\rho}\right)^{\beta}}\left(\frac{\left(T^{\rho}-a^{\rho}\right)^{\beta}\left(T^{\rho}-t^{\rho}\right)}{\rho^{\beta+1} \Gamma(\beta+2)}\right. \\
& \left.-\frac{\mu\left(\xi^{\rho}-a^{\rho}\right)^{\beta+\gamma}\left[(\beta+1)\left(\xi^{\rho}-t^{\rho}\right)-\gamma\left(t^{\rho}-a^{\rho}\right)\right]}{\rho^{\beta+\gamma+1} \Gamma(\beta+\gamma+2)(\beta+1)}\right)\left\{{ }^{\rho} I_{a+}^{\alpha+\beta} h(\eta)-\lambda^{\rho} I_{a+}^{\beta} x(\eta)\right\} .
\end{aligned}
$$

Proof. Applying ${ }^{\rho} I_{a+}^{\alpha}$ on the fractional differential equation in (11) and using Lemma 1 yield:

$$
\left({ }_{c}^{\rho} D_{a+}^{\beta}+\lambda\right) x(t)={ }^{\rho} I_{a+}^{\alpha} h(t)+c_{1}+c_{2} \frac{\left(t^{\rho}-a^{\rho}\right)}{\rho}
$$

for some $c_{1}, c_{2} \in \mathbb{R}$.

Applying ${ }^{\rho} I_{a+}^{\beta}$ to both sides of Equation (13), the general solution of the Langevin equation in (11) is found to be:

$$
x(t)={ }^{\rho} I_{a+}^{\alpha+\beta} h(t)-\lambda^{\rho} I_{a+}^{\beta} x(t)+c_{1} \frac{\left(t^{\rho}-a^{\rho}\right)^{\beta}}{\rho^{\beta} \Gamma(\beta+1)}+c_{2} \frac{\left(t^{\rho}-a^{\rho}\right)^{\beta+1}}{\rho^{\beta+1} \Gamma(\beta+2)}+c_{3},
$$

where $c_{3} \in \mathbb{R}$.

Using the condition $x(a)=0$ in (14), we find that $c_{3}=0$. Inserting the value of $c_{3}$ in (14) and then applying the operator ${ }^{\rho} I_{a+}^{\gamma}$ on the resulting equation, we get:

$$
{ }^{\rho} I_{a}^{\gamma} x(t)={ }^{\rho} I_{a+}^{\alpha+\beta+\gamma} h(t)-\lambda^{\rho} I_{a+}^{\beta+\gamma} x(t)+c_{1} \frac{\left(t^{\rho}-a^{\rho}\right)^{\beta+\gamma}}{\rho^{\beta+\gamma} \Gamma(\beta+\gamma+1)}+c_{2} \frac{\left(t^{\rho}-a^{\rho}\right)^{\beta+\gamma+1}}{\rho^{\beta+\gamma+1} \Gamma(\beta+\gamma+2)} .
$$

Using the boundary conditions $x(\eta)=0$ and $x(T)=\mu^{\rho} I_{a+}^{\gamma} x(\xi)$ together with (14) and (15) leads to a system of algebraic equations in $c_{1}$ and $c_{2}$, which, upon solving, yields:

$$
\begin{aligned}
c_{1}= & \frac{\rho^{\beta} \Gamma(\beta+1)}{\Omega\left(\eta^{\rho}-a^{\rho}\right)^{\beta}}\left\{\frac{\left(\eta^{\rho}-a^{\rho}\right)^{\beta+1}}{\rho^{\beta+1} \Gamma(\beta+2)}\left({ }^{\rho} I_{a+}^{\alpha+\beta} h(T)-\lambda^{\rho} I_{a+}^{\beta} x(T)-\mu^{\rho} I^{\alpha+\beta+\gamma} h(\xi)+\mu \lambda^{\rho} I^{\beta+\gamma} x(\xi)\right)\right. \\
& \left.-\left(\frac{\left(T^{\rho}-a^{\rho}\right)^{\beta+1}}{\rho^{\beta+1} \Gamma(\beta+2)}-\frac{\mu\left(\xi^{\rho}-a^{\rho}\right)^{\beta+\gamma+1}}{\rho^{\beta+\gamma+1} \Gamma(\beta+\gamma+2)}\right)\left({ }^{\rho} I_{a+}^{\alpha+\beta} h(\eta)-\lambda^{\rho} I_{a+}^{\beta} x(\eta)\right)\right\}, \\
c_{2}= & -\frac{\rho^{\beta} \Gamma(\beta+1)}{\Omega\left(\eta^{\rho}-a^{\rho}\right)^{\beta}}\left\{\frac{\left(\eta^{\rho}-a^{\rho}\right)^{\beta}}{\rho^{\beta} \Gamma(\beta+1)}\left({ }^{\rho} I_{a+}^{\alpha+\beta} h(T)-\lambda^{\rho} I_{a+}^{\beta} x(T)-\mu^{\rho} I^{\alpha+\beta+\gamma} h(\xi)+\mu \lambda^{\rho} I^{\beta+\gamma} x(\xi)\right)\right. \\
& \left.-\left(\frac{\left(T^{\rho}-a^{\rho}\right)^{\beta}}{\rho^{\beta} \Gamma(\beta+1)}-\frac{\mu\left(\xi^{\rho}-a^{\rho}\right)^{\beta+\gamma}}{\rho^{\beta+\gamma} \Gamma(\beta+\gamma+1)}\right)\left({ }^{\rho} I_{a+}^{\alpha+\beta} h(\eta)-\lambda^{\rho} I_{a+}^{\beta} x(\eta)\right)\right\} .
\end{aligned}
$$

Inserting the values of $c_{1}, c_{2}$ and $c_{3}$ in (13) yields the solution (12). The converse of the Lemma 2, can be obtained by direct computation. This finishes the proof.

\section{Existence and Uniqueness Results}

In view of Lemma 2, we introduce an operator $\mathcal{F}: \mathcal{C} \rightarrow \mathcal{C}$ by:

$$
\begin{aligned}
\mathcal{F}(x)(t) & ={ }^{\rho} I_{a+}^{\alpha+\beta} f(t, x(t))-\lambda^{\rho} I_{a+}^{\beta} x(t)+\frac{\left(t^{\rho}-a^{\rho}\right)^{\beta}\left(\eta^{\rho}-t^{\rho}\right)}{\rho^{\beta+1} \Gamma(\beta+2) \Omega}\left\{{ }^{\rho} I_{a+}^{\alpha+\beta} f(T, x(T))-\lambda^{\rho} I_{a+}^{\beta} x(T)\right. \\
& \left.-\mu^{\rho} I_{a+}^{\alpha+\beta+\gamma} f(\xi, x(\xi))+\mu \lambda^{\rho} I_{a+}^{\beta+\gamma} x(\xi)\right\}-\frac{\left(t^{\rho}-a^{\rho}\right)^{\beta}}{\Omega\left(\eta^{\rho}-a^{\rho}\right)^{\beta}}\left[\frac{\left(T^{\rho}-a^{\rho}\right)^{\beta}\left(T^{\rho}-t^{\rho}\right)}{\rho^{\beta+1} \Gamma(\beta+2)}\right. \\
& \left.-\frac{\mu\left(\xi^{\rho}-a^{\rho}\right)^{\beta+\gamma}\left[(\beta+1)\left(\xi^{\rho}-t^{\rho}\right)-\gamma\left(t^{\rho}-a^{\rho}\right)\right]}{\rho^{\beta+\gamma+1} \Gamma(\beta+\gamma+2)(\beta+1)}\right]\left\{{ }^{\rho} I_{a+}^{\alpha+\beta} f(\eta, x(\eta))-\lambda^{\rho} I_{a+}^{\beta} x(\eta)\right\} .
\end{aligned}
$$


Here, $\mathcal{C}$ denotes the Banach space of all continuous functions from $[a, T]$ to $\mathbb{R}$ equipped with the norm $\|x\|=\sup _{t \in[a, T]}|x(t)|$.

For the sake of computational convenience, we set:

$$
\begin{aligned}
\Lambda_{1}= & \frac{\left(T^{\rho}-a^{\rho}\right)^{\alpha+\beta}}{\rho^{\alpha+\beta} \Gamma(\alpha+\beta+1)}\left[1+\frac{\zeta_{1}}{\rho^{\beta+1} \Gamma(\beta+2)|\Omega|}\right]+\frac{|\mu|\left(\xi^{\rho}-a^{\rho}\right)^{\alpha+\beta+\gamma} \zeta_{1}}{\rho^{\alpha+2 \beta+\gamma+1} \Gamma(\alpha+\beta+\gamma+1) \Gamma(\beta+2)|\Omega|} \\
& +\frac{\left(\eta^{\rho}-a^{\rho}\right)^{\alpha} \zeta_{2}}{\rho^{\alpha+\beta} \Gamma(\alpha+\beta+1)|\Omega|}, \\
\Lambda_{2}= & \frac{|\lambda|\left(T^{\rho}-a^{\rho}\right)^{\beta}}{\rho^{\beta} \Gamma(\beta+1)}\left[1+\frac{\zeta_{1}}{\rho^{\beta+1} \Gamma(\beta+2)|\Omega|}\right]+\frac{|\mu||\lambda|\left(\xi^{\rho}-a^{\rho}\right)^{\beta+\gamma} \zeta_{1}}{\rho^{2 \beta+\gamma+1} \Gamma(\beta+\gamma+1) \Gamma(\beta+2)|\Omega|} \\
& \quad+\frac{|\lambda| \zeta_{2}}{\rho^{\beta} \Gamma(\beta+1)|\Omega|},
\end{aligned}
$$

where:

$$
\begin{gathered}
\zeta_{1}:=\max _{t \in[a, T]}\left|\left(t^{\rho}-a^{\rho}\right)^{\beta}\left(\eta^{\rho}-t^{\rho}\right)\right| \\
\zeta_{2}:=\max _{t \in[a, T]}\left|\left(t^{\rho}-a^{\rho}\right)^{\beta}\left[\frac{\left(T^{\rho}-a^{\rho}\right)^{\beta}\left(T^{\rho}-t^{\rho}\right)}{\rho^{\beta+1} \Gamma(\beta+2)}-\frac{\mu\left(\xi^{\rho}-a^{\rho}\right)^{\beta+\gamma}\left[(\beta+1)\left(\xi^{\rho}-t^{\rho}\right)-\gamma\left(t^{\rho}-a^{\rho}\right)\right]}{\rho^{\beta+\gamma+1} \Gamma(\beta+\gamma+2)(\beta+1)}\right]\right| .
\end{gathered}
$$

Now, we are in a position to present our main results. Our first existence result for the problem (1) is based on Krasnoselskii's fixed point theorem [25], which is stated below.

Lemma 3. (Krasnoselskii's fixed point theorem) Let $\mathcal{S}$ be a closed convex and non-empty subset of a Banach space E. Let $\mathcal{G}_{1}, \mathcal{G}_{2}$ be the operators from $\mathcal{S}$ to $E$ such that $(a) \mathcal{G}_{1} x+\mathcal{G}_{2} y \in \mathcal{S}$ whenever $u, v \in \mathcal{S} ;(b) \mathcal{G}_{1}$ is compact and continuous; and $(c) \mathcal{G}_{2}$ is a contraction mapping. Then, there exists a fixed point $\omega \in \mathcal{S}$ such that $\omega=\mathcal{G}_{1} \omega+\mathcal{G}_{2} \omega$.

Theorem 1. Let $f: J \times \mathbb{R} \rightarrow \mathbb{R}$ be a continuous function such that the following condition holds:

$\left(A_{1}\right)$ There exists a continuous function $\phi \in C\left([a, T], \mathbb{R}^{+}\right)$such that:

$$
|f(t, u)| \leq \phi(t), \quad \forall(t, u) \in J \times \mathbb{R} .
$$

Then, the problem (1) has at least one solution on J, provided that:

$$
\Lambda_{2}<1 \text {. }
$$

Proof. Introduce a closed ball $B_{r}=\{x \in \mathcal{C}:\|x\| \leq r\}$, with $r>\frac{\|\phi\| \Lambda_{1}}{1-\Lambda_{2}},\|\phi\|=\sup _{t \in[a, T]}|\phi(t)|$, where $\Lambda_{2}$ is given by (18). Then, we define operators $\mathcal{F}_{1}$ and $\mathcal{F}_{2}$ from $B_{r}$ to $\mathcal{C}$ by:

$$
\begin{aligned}
\mathcal{F}_{1}(x)(t)= & { }^{\rho} I_{a+}^{\alpha+\beta} f(t, x(t))+\frac{\left(t^{\rho}-a^{\rho}\right)^{\beta}\left(\eta^{\rho}-t^{\rho}\right)}{\rho^{\beta+1} \Gamma(\beta+2) \Omega}\left\{{ }^{\rho} I_{a+}^{\alpha+\beta} f(T, x(T))-\mu^{\rho} I_{a+}^{\alpha+\beta+\gamma} f(\xi, x(\xi))\right\} \\
& -\frac{\left(t^{\rho}-a^{\rho}\right)^{\beta}}{\Omega\left(\eta^{\rho}-a^{\rho}\right)^{\beta}}\left[\frac{\left(T^{\rho}-a^{\rho}\right)^{\beta}\left(T^{\rho}-t^{\rho}\right)}{\rho^{\beta+1} \Gamma(\beta+2)}-\frac{\mu\left(\xi^{\rho}-a^{\rho}\right)^{\beta+\gamma}\left[(\beta+1)\left(\xi^{\rho}-t^{\rho}\right)-\gamma\left(t^{\rho}-a^{\rho}\right)\right]}{\rho^{\beta+\gamma+1} \Gamma(\beta+\gamma+2)(\beta+1)}\right] \\
& \times{ }^{\rho} I_{a+}^{\alpha+\beta} f(\eta, x(\eta)), \\
\mathcal{F}_{2}(x)(t)= & -\lambda^{\rho} I_{a+}^{\beta} x(t)-\frac{\left(t^{\rho}-a^{\rho}\right)^{\beta}\left(\eta^{\rho}-t^{\rho}\right)}{\rho^{\beta+1} \Gamma(\beta+2) \Omega}\left\{\lambda^{\rho} I_{a+}^{\beta} x(T)-\mu \lambda^{\rho} I_{a+}^{\beta+\gamma} x(\xi)\right\}+\frac{\lambda\left(t^{\rho}-a^{\rho}\right)^{\beta}}{\Omega\left(\eta^{\rho}-a^{\rho}\right)^{\beta}} \times \\
& \times\left[\frac{\left(T^{\rho}-a^{\rho}\right)^{\beta}\left(T^{\rho}-t^{\rho}\right)}{\rho^{\beta+1} \Gamma(\beta+2)}-\frac{\mu\left(\xi^{\rho}-a^{\rho}\right)^{\beta+\gamma}\left[(\beta+1)\left(\xi^{\rho}-t^{\rho}\right)-\gamma\left(t^{\rho}-a^{\rho}\right)\right]}{\rho^{\beta+\gamma+1} \Gamma(\beta+\gamma+2)(\beta+1)}\right]^{\rho} I_{a+}^{\beta} x(\eta) .
\end{aligned}
$$


Note that $\mathcal{F}=\mathcal{F}_{1}+\mathcal{F}_{2}$ on $B_{r}$. For $x, y \in B_{r}$, we find that:

$$
\begin{aligned}
& \left\|\mathcal{F}_{1} x+\mathcal{F}_{2} y\right\| \\
& \leq \sup _{t \in J}\left\{{ }^{\rho} I_{a+}^{\alpha+\beta}|f(t, x(t))|+|\lambda|^{\rho} I_{a+|y(t)|}^{\beta} \mid\right. \\
& +\frac{\left(t^{\rho}-a^{\rho}\right)^{\beta}\left|\left(\eta^{\rho}-t^{\rho}\right)\right|}{\rho^{\beta+1} \Gamma(\beta+2)|\Omega|}\left\{\rho I_{a+}^{\alpha+\beta}|f(T, x(T))|+|\lambda|^{\rho} I_{a+}^{\beta}|y(T)|\right. \\
& \left.+|\mu|^{\rho} I_{a+}^{\alpha+\beta+\gamma}|f(\xi, x(\xi))|+|\mu||\lambda|^{\rho} I_{a+}^{\beta+\gamma}|x(\xi)|\right\}+\frac{\left(t^{\rho}-a^{\rho}\right)^{\beta}}{|\Omega|\left(\eta^{\rho}-a^{\rho}\right)^{\beta}} \mid \frac{\left(T^{\rho}-a^{\rho}\right)^{\beta}\left(T^{\rho}-t^{\rho}\right)}{\rho^{\beta+1} \Gamma(\beta+2)} \\
& -\frac{\mu\left(\xi^{\rho}-a^{\rho}\right)^{\beta+\gamma}\left[(\beta+1)\left(\xi^{\rho}-t^{\rho}\right)-\gamma\left(t^{\rho}-a^{\rho}\right)\right]}{\rho^{\beta+\gamma+1} \Gamma(\beta+\gamma+2)(\beta+1)} \mid\left\{\rho I_{a+}^{\alpha+\beta}|f(\eta, x(\eta))|+|\lambda|^{\rho} I_{a+}^{\beta}|y(\eta)|\right\} \\
& \leq\|\phi\|\left\{\frac{\left(T^{\rho}-a^{\rho}\right)^{\alpha+\beta}}{\rho^{\alpha+\beta} \Gamma(\alpha+\beta+1)}\left[1+\frac{\zeta_{1}}{\rho^{\beta+1} \Gamma(\beta+2)|\Omega|}\right]+\frac{|\mu|\left(\xi^{\rho}-a^{\rho}\right)^{\alpha+\beta+\gamma} \zeta_{1}}{\rho^{\alpha+2 \beta+\gamma+1} \Gamma(\alpha+\beta+\gamma+1) \Gamma(\beta+2)|\Omega|}\right. \\
& \left.+\frac{\left(\eta^{\rho}-a^{\rho}\right)^{\alpha} \zeta_{2}}{\rho^{\alpha+\beta} \Gamma(\alpha+\beta+1)|\Omega|}\right\}+\|x\|\left\{\frac{|\lambda|\left(T^{\rho}-a^{\rho}\right)^{\beta}}{\rho^{\beta} \Gamma(\beta+1)}\left[1+\frac{\zeta_{1}}{\rho^{\beta+1} \Gamma(\beta+2)|\Omega|}\right]\right. \\
& \left.+\frac{|\mu||\lambda|\left(\xi^{\rho}-a^{\rho}\right)^{\beta+\gamma} \zeta_{1}}{\rho^{2 \beta+\gamma+1} \Gamma(\beta+\gamma+1) \Gamma(\beta+2)|\Omega|}+\frac{|\lambda| \zeta_{2}}{\rho^{\beta} \Gamma(\beta+1)|\Omega|}\right\} \\
& \leq\|\phi\| \Lambda_{1}+r \Lambda_{2}<r \text {. }
\end{aligned}
$$

Thus, $\mathcal{F}_{1} x+\mathcal{F}_{2} y \in B_{r}$.

Next, it will be shown that $\mathcal{F}_{2}$ is a contraction. For that, let $x, y \in \mathcal{C}$. Then:

$$
\begin{aligned}
& \left\|\mathcal{F}_{2} x-\mathcal{F}_{2} y\right\| \\
\leq & \sup _{t \in J}\left\{\left.|\lambda|\right|^{\rho} I_{a+}^{\beta}|x(t)-y(t)|+\frac{\left|\left(t^{\rho}-a^{\rho}\right)^{\beta}\left(\eta^{\rho}-t^{\rho}\right)\right|}{\rho^{\beta+1} \Gamma(\beta+2)|\Omega|} \times\right. \\
& \times\left\{|\lambda|^{\rho} I_{a+}^{\beta}|x(T)-y(T)|+|\mu||\lambda|^{\rho} I_{a+}^{\beta+\gamma}|x(\xi)-y(\xi)|\right\} \\
& +\frac{|\lambda|\left|\left(t^{\rho}-a^{\rho}\right)^{\beta}\right|}{|\Omega|\left(\eta^{\rho}-a^{\rho}\right)^{\beta} \mid}\left|\frac{\left(T^{\rho}-a^{\rho}\right)^{\beta}\left(T^{\rho}-t^{\rho}\right)}{\rho^{\beta+1} \Gamma(\beta+2)}-\frac{\mu\left(\xi^{\rho}-a^{\rho}\right)^{\beta+\gamma}\left[(\beta+1)\left(\xi^{\rho}-t^{\rho}\right)-\gamma\left(t^{\rho}-a^{\rho}\right)\right]}{\rho^{\beta+\gamma+1} \Gamma(\beta+\gamma+2)(\beta+1)}\right| \times \\
& \left.\times{ }^{\rho} I_{a+}^{\beta}|x(\eta)-y(\eta)|\right\} \\
\leq & \left\{\frac{|\lambda|\left(T^{\rho}-a^{\rho}\right)^{\beta}}{\rho^{\beta} \Gamma(\beta+1)}\left[1+\frac{|\mu||\lambda|\left(\xi^{\rho}-a^{\rho}\right)^{\beta+\gamma} \zeta_{1}}{\rho^{\beta+1} \Gamma(\beta+2)|\Omega|}\right]+\frac{\mid \zeta_{1}}{\rho^{2 \beta+\gamma+1} \Gamma(\beta+\gamma+1) \Gamma(\beta+2)|\Omega|}\right. \\
& \left.+\frac{|\lambda| \zeta_{2}}{\rho^{\beta} \Gamma(\beta+1)|\Omega|}\right\}\|x-y\| \\
= & \Lambda_{2}\|x-y\|,
\end{aligned}
$$

which, by the condition (21), implies that $\mathcal{F}_{2}$ is a contraction. The continuity of the operator $\mathcal{F}_{1}$ follows from that of $f$. Furthermore, $\mathcal{F}_{1}$ is uniformly bounded on $B_{r}$ as:

$$
\left\|\mathcal{F}_{1} x\right\| \leq\|\phi\| \Lambda_{1}
$$

Finally, we establish the compactness of the operator $\mathcal{F}_{1}$. Let us set $\sup _{(t, x) \in J \times B_{r}}|f(t, x)|=\bar{f}<\infty$. Then, for $t_{1}, t_{2} \in J, t_{1}<t_{2}$, we have:

$$
\left|\left(\mathcal{F}_{1} x\right)\left(t_{2}\right)-\left(\mathcal{F}_{1} x\right)\left(t_{1}\right)\right|
$$




$$
\begin{aligned}
= & \mid \frac{\rho^{1-(\alpha+\beta)}}{\Gamma(\alpha+\beta)}\left[\int_{0}^{t_{1}} s^{\rho-1}\left[\left(t_{2}^{\rho}-s^{\rho}\right)^{\alpha+\beta-1}-\left(t_{1}^{\rho}-s^{\rho}\right)^{\alpha+\beta-1}\right] f(s, x(s)) d s\right. \\
& \left.+\int_{t_{1}}^{t_{2}} s^{\rho-1}\left(t_{2}^{\rho}-s^{\rho}\right)^{\alpha+\beta-1} f(s, x(s)) d s\right] \\
& +\left[\frac{\left(t_{2}^{\rho}-a^{\rho}\right)^{\beta}\left(\eta^{\rho}-t_{2}^{\rho}\right)}{\rho^{\beta+1} \Gamma(\beta+2) \Omega}-\frac{\left(t_{1}^{\rho}-a^{\rho}\right)^{\beta}\left(\eta^{\rho}-t_{1}^{\rho}\right)}{\rho^{\beta+1} \Gamma(\beta+2) \Omega}\right]\left\{{ }^{\rho} I_{a+}^{\alpha+\beta} f(T, x(T))-\mu^{\rho} I_{a+}^{\alpha+\beta+\gamma} f(\xi, x(\xi))\right\} \\
& -\left[\frac{\left(t_{2}^{\rho}-a^{\rho}\right)^{\beta}}{\Omega\left(\eta^{\rho}-a^{\rho}\right)^{\beta}}\left[\frac{\left(T^{\rho}-a^{\rho}\right)^{\beta}\left(T^{\rho}-t_{2}^{\rho}\right)}{\rho^{\beta+1} \Gamma(\beta+2)}-\frac{\mu\left(\xi^{\rho}-a^{\rho}\right)^{\beta+\gamma}\left[(\beta+1)\left(\xi^{\rho}-t_{2}^{\rho}\right)-\gamma\left(t^{\rho}-a^{\rho}\right)\right]}{\rho^{\beta+\gamma+1} \Gamma(\beta+\gamma+2)(\beta+1)}\right]\right. \\
& \left.-\frac{\left(t_{1}^{\rho}-a^{\rho}\right)^{\beta}}{\Omega\left(\eta^{\rho}-a^{\rho}\right)^{\beta}}\left[\frac{\left(T^{\rho}-a^{\rho}\right)^{\beta}\left(T^{\rho}-t_{1}^{\rho}\right)}{\rho^{\beta+1} \Gamma(\beta+2)}-\frac{\mu\left(\xi^{\rho}-a^{\rho}\right)^{\beta+\gamma}\left[(\beta+1)\left(\xi^{\rho}-t_{1}^{\rho}\right)-\gamma\left(t_{1}^{\rho}-a^{\rho}\right)\right]}{\rho^{\beta+\gamma+1} \Gamma(\beta+\gamma+2)(\beta+1)}\right]\right] \times \\
& \times{ }^{\rho} I_{a+\beta}^{\alpha+\beta} f(\eta, x(\eta)) \mid \\
\leq & \frac{\bar{f}}{\rho^{\alpha+\beta} \Gamma(\alpha+\beta+1)}\left\{\left|t_{2}^{\rho(\alpha+\beta)}-t_{1}^{\rho(\alpha+\beta)}\right|+2\left(t_{2}^{\rho}-t_{1}^{\rho}\right)^{\alpha+\beta}\right\} \\
& +\left|\frac{\left(t_{2}^{\rho}-a^{\rho}\right)^{\beta}\left(\eta^{\rho}-t_{2}^{\rho}\right)}{\rho^{\beta+1} \Gamma(\beta+2) \Omega}-\frac{\left(t_{1}^{\rho}-a^{\rho}\right)^{\beta}\left(\eta^{\rho}-t_{1}^{\rho}\right)}{\rho^{\beta+1} \Gamma(\beta+2) \Omega}\right|\left\{{ }^{\rho} I_{a+}^{\alpha+\beta}|f(T, x(T))|+\mu^{\rho} I_{a+}^{\alpha+\beta+\gamma}|f(\xi, x(\xi))|\right\} \\
& +\mid \frac{\left(t_{2}^{\rho}-a^{\rho}\right)^{\beta}}{\Omega\left(\eta^{\rho}-a^{\rho}\right)^{\beta}}\left[\frac{\left(T^{\rho}-a^{\rho}\right)^{\beta}\left(T^{\rho}-t_{2}^{\rho}\right)}{\rho^{\beta+1} \Gamma(\beta+2)}-\frac{\mu\left(\xi^{\rho}-a^{\rho}\right)^{\beta+\gamma}\left[(\beta+1)\left(\xi^{\rho}-t_{2}^{\rho}\right)-\gamma\left(t_{2}^{\rho}-a^{\rho}\right)\right]}{\rho^{\beta+\gamma+1} \Gamma(\beta+\gamma+2)(\beta+1)}\right] \\
& -\frac{\left(t_{1}^{\rho}-a^{\rho}\right)^{\beta}}{\Omega\left(\eta^{\rho}-a^{\rho}\right)^{\beta}}\left[\frac{\left(T^{\rho}-a^{\rho}\right)^{\beta}\left(T^{\rho}-t_{1}^{\rho}\right)}{\rho^{\beta+1} \Gamma(\beta+2)}-\frac{\mu\left(\xi^{\rho}-a^{\rho}\right)^{\beta+\gamma}\left[(\beta+1)\left(\xi^{\rho}-t_{1}^{\rho}\right)-\gamma\left(t_{1}^{\rho}-a^{\rho}\right)\right]}{\rho^{\beta+\gamma+1} \Gamma(\beta+\gamma+2)(\beta+1)}\right] \mid \times \\
& \times I_{a+}^{\alpha+\beta}|f(\eta, x(\eta))|,
\end{aligned}
$$

which tends to zero as $t_{2} \rightarrow t_{1}$, independently of $x \in B_{r}$. Thus, $\mathcal{F}_{1}$ is equicontinuous. Therefore, $\mathcal{F}_{1}$ is relatively compact on $B_{r}$. As a consequence, we deduce by the the Arzelá-Ascoli theorem that $\mathcal{F}_{1}$ is compact on $B_{r}$. Thus, the hypothesis of Lemma 3 is satisfied. Therefore, the conclusion of Lemma 3 applies, and hence, there exists at least one solution for the problem (1) on $J$.

In the next result, the uniqueness of solutions for the problem (1) is shown by means of the Banach contraction mapping principle.

Theorem 2. Let $f: J \times \mathbb{R} \rightarrow \mathbb{R}$ be a continuous function satisfying the Lipschitz condition:

$$
|f(t, u)-f(t, v)| \leq L|u-v|, L>0, /, \quad \text { for } t \in J \text { and every } u, v \in \mathbb{R} .
$$

Then, there exists a unique solution for the problem (1) on $[a, T]$, provided that:

$$
L \Lambda_{1}+\Lambda_{2}<1,
$$

where $\Lambda_{1}$ and $\Lambda_{2}$ are respectively given by (17) and (18).

Proof. In the first step, we show that $\mathcal{F} B_{\bar{r}} \subset B_{\bar{r}}$, where $B_{\bar{r}}=\{x \in C([a, T], \mathbb{R}):\|x\| \leq \bar{r}\}, M=$ $\sup _{t \in[a, T]}|f(t, 0)|, \bar{r} \geq \frac{\Lambda_{1} M}{1-L \Lambda_{1}-\Lambda_{2}}$, and the operator $\mathcal{F}: \mathcal{C} \rightarrow \mathcal{C}$ is given by (16). For $x \in B_{\bar{r}}$, using $\left(A_{2}\right)$, we get:

$$
\begin{aligned}
& |\mathcal{F}(x)(t)| \\
\leq & { }^{\rho} I_{a+}^{\alpha+\beta}[|f(t, x(t))-f(t, 0)|+|f(t, 0)|]+|\lambda|{ }^{\rho} I_{a+}^{\beta}|x(t)| \\
& +\frac{\left(t^{\rho}-a^{\rho}\right)^{\beta}\left|\left(\eta^{\rho}-t^{\rho}\right)\right|}{\rho^{\beta+1} \Gamma(\beta+2)|\Omega|}\left\{{ }^{\rho} I_{a+}^{\alpha+\beta}[|f(T, x(T))-f(T, 0)|+|f(T, 0)|]+|\lambda|^{\rho} I_{a+}^{\beta}|x(T)|\right. \\
& \left.+|\mu|^{\rho} I_{a+}^{\alpha+\beta+\gamma}[|f(\xi, x(\xi))-f(\xi, 0)|+|f(\xi, 0)|]+|\mu||\lambda|^{\rho} I_{a+}^{\beta+\gamma}|x(\xi)|\right\}
\end{aligned}
$$




$$
\begin{aligned}
& +\frac{\left(t^{\rho}-a^{\rho}\right)^{\beta}}{|\Omega|\left(\eta^{\rho}-a^{\rho}\right)^{\beta}}\left|\frac{\left(T^{\rho}-a^{\rho}\right)^{\beta}\left(T^{\rho}-t^{\rho}\right)}{\rho^{\beta+1} \Gamma(\beta+2)}-\frac{\mu\left(\xi^{\rho}-a^{\rho}\right)^{\beta+\gamma}\left[(\beta+1)\left(\xi^{\rho}-t^{\rho}\right)-\gamma\left(t^{\rho}-a^{\rho}\right)\right]}{\rho^{\beta+\gamma+1} \Gamma(\beta+\gamma+2)(\beta+1)}\right| \\
& \times\left\{{ }^{\rho} I_{a+}^{\alpha+\beta}[|f(\eta, x(\eta))-f(\eta, 0)|+|f(\eta, 0)|]+|\lambda|^{\rho} I_{a+}^{\beta}|x(\eta)|\right\} \\
\leq \quad & (L \bar{r}+M)\left(\frac{\left(T^{\rho}-a^{\rho}\right)^{\alpha+\beta}}{\rho^{\alpha+\beta} \Gamma(\alpha+\beta+1)}\left[1+\frac{\zeta_{1}}{\rho^{\beta+1} \Gamma(\beta+2)|\Omega|}\right]+\frac{|\mu|\left(\xi^{\rho}-a^{\rho}\right)^{\alpha+\beta+\gamma} \zeta_{1}}{\rho^{\alpha+2 \beta+\gamma+1} \Gamma(\alpha+\beta+\gamma+1) \Gamma(\beta+2)|\Omega|}\right. \\
& \left.+\frac{\left(\eta^{\rho}-a^{\rho}\right)^{\alpha} \zeta_{2}}{\rho^{\alpha+\beta} \Gamma(\alpha+\beta+1)|\Omega|}\right) \\
& +\bar{r}\left(\frac{|\lambda|\left(T^{\rho}-a^{\rho}\right)^{\beta}}{\rho^{\beta} \Gamma(\beta+1)}\left[1+\frac{\zeta_{1}}{\rho^{\beta+1} \Gamma(\beta+2)|\Omega|}\right]+\frac{|\mu||\lambda|\left(\xi^{\rho}-a^{\rho}\right)^{\beta+\gamma} \zeta_{1}}{\rho^{2 \beta+\gamma+1} \Gamma(\beta+\gamma+1) \Gamma(\beta+2)|\Omega|}+\frac{|\lambda| \zeta_{2}}{\rho^{\beta} \Gamma(\beta+1)|\Omega|}\right) \\
= & (L \bar{r}+M) \Lambda_{1}+\Lambda_{2} \bar{r} \leq \bar{r},
\end{aligned}
$$

which, on taking the norm for $t \in[a, T]$, implies that $\|\mathcal{F}(x)\| \leq \bar{r}$. Thus, the operator $\mathcal{F}$ maps $B_{\bar{r}}$ into itself. Now, we proceed to prove that the operator $\mathcal{F}$ is a contraction. For $x, y \in C([a, T], \mathbb{R})$ and $t \in[a, T]$, we have:

$$
\begin{aligned}
& |\mathcal{F}(x)(t)-\mathcal{F}(y)(t)| \\
& \leq{ }^{\rho} I_{a+}^{\alpha+\beta}|f(t, x(t))-f(t, y(t))|+|\lambda|^{\rho} I_{a+}^{\beta}|x(t)-y(t)| \\
& +\frac{\left(t^{\rho}-a^{\rho}\right)^{\beta}\left|\left(\eta^{\rho}-t^{\rho}\right)\right|}{\rho^{\beta+1} \Gamma(\beta+2)|\Omega|}\left\{{ }^{\rho} I_{a+}^{\alpha+\beta}|f(T, x(T))-f(T, y(T))|+|\lambda|^{\rho} I_{a+}^{\beta}|x(T)-y(T)|\right. \\
& \left.+|\mu|^{\rho} I_{a+}^{\alpha+\beta+\gamma}|f(\xi, x(\xi))-f(\xi, y(\xi))|+|\mu||\lambda|^{\rho} I_{a+}^{\beta+\gamma}|x(\xi)-y(\xi)|\right\} \\
& +\frac{\left(t^{\rho}-a^{\rho}\right)^{\beta}}{|\Omega|\left(\eta^{\rho}-a^{\rho}\right)^{\beta}}\left|\frac{\left(T^{\rho}-a^{\rho}\right)^{\beta}\left(T^{\rho}-t^{\rho}\right)}{\rho^{\beta+1} \Gamma(\beta+2)}-\frac{\mu\left(\xi^{\rho}-a^{\rho}\right)^{\beta+\gamma}\left[(\beta+1)\left(\xi^{\rho}-t^{\rho}\right)-\gamma\left(t^{\rho}-a^{\rho}\right)\right]}{\rho^{\beta+\gamma+1} \Gamma(\beta+\gamma+2)(\beta+1)}\right| \times \\
& \times\left\{{ }^{\rho} I_{a+}^{\alpha+\beta}|f(\eta, x(\eta))-f(\eta, y(\eta))|+|\lambda|^{\rho} I_{a+}^{\beta}|x(\eta)-y(\eta)|\right\} \\
& \leq L\|x-y\|\left(\frac{\left(T^{\rho}-a^{\rho}\right)^{\alpha+\beta}}{\rho^{\alpha+\beta} \Gamma(\alpha+\beta+1)}\left[1+\frac{\zeta_{1}}{\rho^{\beta+1} \Gamma(\beta+2)|\Omega|}\right]\right. \\
& +\frac{|\mu|\left(\xi^{\rho}-a^{\rho}\right)^{\alpha+\beta+\gamma} \zeta_{1}}{\rho^{\alpha+2 \beta+\gamma+1} \Gamma(\alpha+\beta+\gamma+1) \Gamma(\beta+2)|\Omega|} \\
& \left.+\frac{\left(\eta^{\rho}-a^{\rho}\right)^{\alpha} \zeta_{2}}{\rho^{\alpha+\beta} \Gamma(\alpha+\beta+1)|\Omega|}\right)+\|x-y\|\left(\frac{|\lambda|\left(T^{\rho}-a^{\rho}\right)^{\beta}}{\rho^{\beta} \Gamma(\beta+1)}\left[1+\frac{\zeta_{1}}{\rho^{\beta+1} \Gamma(\beta+2)|\Omega|}\right]\right. \\
& \left.+\frac{|\mu||\lambda|\left(\xi^{\rho}-a^{\rho}\right)^{\beta+\gamma} \zeta_{1}}{\rho^{2 \beta+\gamma+1} \Gamma(\beta+\gamma+1) \Gamma(\beta+2)|\Omega|}+\frac{|\lambda| \zeta_{2}}{\rho^{\beta} \Gamma(\beta+1)|\Omega|}\right) \\
& =\left(L \Lambda_{1}+\Lambda_{2}\right)\|x-y\| \text {. }
\end{aligned}
$$

Taking the norm of the above inequality for $t \in[a, T]$, we get:

$$
\|\mathcal{F}(x)-\mathcal{F}(y)\| \leq\left(L \Lambda_{1}+\Lambda_{2}\right)\|x-y\|,
$$

which implies that the operator $\mathcal{F}$ is a contraction on account of the condition (22). Thus, we deduce by the Banach contraction mapping principle that the operator $\mathcal{F}$ has a unique fixed point. Hence, there exists a unique solution for the problem (1). The proof is complete.

Example 1. Let us consider the following boundary value problem:

$$
\left\{\begin{array}{l}
{ }_{c}^{1 / 3} D^{5 / 4}\left({ }_{c}^{1 / 3} D^{1 / 4}+1 / 5\right) x(t)=\frac{1}{\sqrt{400+t}}\left(\frac{|x(t)|+2}{|x(t)|+1}+e^{-t}\right), \quad t \in J:=[1,2] \\
x(1)=0, \quad x(3 / 2)=0, \quad x(2)=2 / 7^{1 / 3} I^{3 / 4} x(7 / 4)
\end{array}\right.
$$


Here, $\rho=1 / 3, \alpha=5 / 4, \beta=1 / 4, \gamma=3 / 4, \lambda=1 / 5, \mu=2 / 7, a=1, \eta=3 / 2, \xi=7 / 4, T=2$ and $f(t, x)=\frac{1}{\sqrt{400+t}}\left(\frac{|x|+2}{|x|+1}+e^{-t}\right)$. Using the given data, we find that $|\Omega| \approx 0.293634, \Lambda_{1} \approx$ $1.336009, \Lambda_{2} \approx 0.673563, \zeta_{1} \approx 0.082260, \zeta_{2} \approx 0.232036$, where $\Omega, \Lambda_{1}, \Lambda_{2}, \zeta_{1}$, and $\zeta_{2}$ are given by $(10)$, (17), (18), (19) and (20) respectively.

For illustrating Theorem 1, we show that all the conditions of Theorem 1 are satisfied. Clearly, $f(t, x)$ is continuous and satisfies the condition $\left(A_{1}\right)$ with $\phi(t)=\frac{2+e^{-t}}{\sqrt{400+t}}$. Furthermore, $\Lambda_{2} \approx$ $0.673563<1$. Thus, all the conditions of Theorem 1 are satisfied, and consequently, the problem (23) has at least one solution on $[1,2]$.

Furthermore, Theorem 2 is applicable to the problem (23) with $L=1 / 20$ as $L \Lambda_{1}+\Lambda_{2} \approx$ $0.740363<1$. Thus, all the assumptions of Theorem 2 are satisfied. Therefore, the conclusion of Theorem 2 applies to the problem (23) on $[1,2]$.

\section{Conclusions}

We have introduced a new type of nonlinear Langevin equation in terms of Liouville-Caputo-type generalized fractional differential operators of different orders and solved it with nonlocal generalized integral boundary conditions. The existence result was obtained by applying the Krasnoselskii fixed point theorem without requiring the nonlinear function to be of the Lipschitz type, while the uniqueness of solutions for the given problem was based on a celebrated fixed point theorem due to Banach. Here, we remark that many known existence results, obtained by means of the Krasnoselskii fixed point theorem, demand the associated nonlinear function to satisfy the Lipschitz condition. Moreover, by fixing the parameters involved in the given problem, we can obtain some new results as special cases of the ones presented in this paper. For example, letting $\rho=1, \mu=0, a=0$ and $T=1$ in the results of Section 3, we get the ones derived in [15].

Author Contributions: Formal analysis, B.A., M.A., A.A., H.M.S. and S.K.N.

Funding: This project was funded by the Deanship of Scientific Research (DSR), King Abdulaziz University, Jeddah, Saudi Arabia, under Grant No. KEP-PhD-24-130-40. The authors acknowledge with thanks the DSR's technical and financial support. The authors also acknowledge the reviewers for their constructive remarks on our work.

Conflicts of Interest: The authors declare no conflict of interest.

\section{References}

1. Zaslavsky, G.M. Hamiltonian Chaos and Fractional Dynamics; Oxford University Press: Oxford, UK, 2005.

2. Magin, R.L. Fractional Calculus in Bioengineering; Begell House Publishers: Danbury, CT, USA, 2006.

3. Kilbas, A.A.; Srivastava, H.M.; Trujillo, J.J. Theory and Applications of Fractional Differential Equations; North-Holland Mathematics Studies, 204; Elsevier Science B.V.: Amsterdam, The Netherlands, 2006.

4. Diethelm, K. The Analysis of Fractional Differential Equations. An Application-Oriented Exposition Using Differential Operators of Liouville-Caputo Type. Lecture Notes in Mathematics 2004; Springer: Berlin, Germany, 2010.

5. Javidi, M.; Ahmad, B. Dynamic analysis of time fractional order phytoplankton-toxic phytoplanktonzooplankton system. Ecol. Model. 2015, 318, 8-18. [CrossRef]

6. Fallahgoul, H.A.; Focardi, S.M.; Fabozzi, F.J. Fractional Calculus and Fractional Processes with Applications to Financial Economics. Theory and Application; Elsevier/Academic Press: London, UK, 2017.

7. Ahmad, B.; Alsaedi, A.; Ntouyas, S.K.; Tariboon, J. Hadamard-Type Fractional Differential Equations, Inclusions and Inequalities; Springer: Cham, Switzerland, 2017.

8. West, B.J.; Picozzi, S. Fractional Langevin model of memory in financial time series. Phys. Rev. E 2002, 65, 037106. [CrossRef] [PubMed]

9. Vinales, A.D.; Desposito, M.A. Anomalous diffusion: Exact solution of the generalized Langevin equation for harmonically bounded particle. Phys. Rev. E 2006, 73, 016111. [CrossRef] [PubMed]

10. Hohenberg, P.C.; Halperin, B.I. Theory of dynamic critical phenomena. Rev. Mod. Phys. 1977, 49, 435-479. [CrossRef] 
11. Metiu, H.; Schon, G. Description of Quantum noise by a Langevin equation. Phys. Rev. Lett. 1984, 53, 13. [CrossRef]

12. Datsko, B.; Gafiychuk, V. Complex nonlinear dynamics in subdiffusive activator-inhibitor systems. Commun. Nonlinear Sci. Numer. Simul. 2012, 17, 1673-1680. [CrossRef]

13. Datsko, B.; Gafiychuk, V. Complex spatio-temporal solutions in fractional reaction-diffusion systems near a bifurcation point. Fract. Calc. Appl. Anal. 2018, 21, 237-253. [CrossRef]

14. Fa, K.S. Fractional Langevin equation and Riemann-Liouville fractional derivative. Eur. Phys. J. E 2007, 24, 139-143.

15. Ahmad, B.; Nieto, J.J.; Alsaedi, A.; El-Shahed, M. A study of nonlinear Langevin equation involving two fractional orders in different intervals. Nonlinear Anal. Real World Appl. 2012, 13, 599-606. [CrossRef]

16. Wang, G.; Zhang, L.; Song, G. Boundary value problem of a nonlinear Langevin equation with two different fractional orders and impulses. Fixed Point Theory Appl. 2012, 2012, 200. [CrossRef]

17. Ahmad, B.; Ntouyas, S.K. New existence results for differential inclusions involving Langevin equation with two indices. J. Nonlinear Convex Anal. 2013, 14, 437-450.

18. Muensawat, T.; Ntouyas, S.K.; Tariboon, J. Systems of generalized Sturm-Liouville and Langevin fractional differential equations. Adv. Differ. Equ. 2017, 2017, 63. [CrossRef]

19. Fazli, H.; Nieto, J.J. Fractional Langevin equation with anti-periodic boundary conditions. Chaos Solitons Fractals 2018, 114, 332-337. [CrossRef]

20. Ahmad, B.; Alsaedi, A.; Salem, S. On a nonlocal integral boundary value problem of nonlinear Langevin equation with different fractional orders. Adv. Differ. Equ. 2019, 2019, 57. [CrossRef]

21. Laskin, N. Fractional quantum mechanics and Levy path integrals. Phys. Lett. A 2000, 268, 298-305. [CrossRef]

22. Katugampola, U.N. New Approach to a generalized fractional integral. Appl. Math. Comput. 2015, 218, 860-865. [CrossRef]

23. Katugampola, U.N. A new approach to generalized fractional derivatives. Bull. Math. Anal. Appl. 2014, 6, $1-15$.

24. Jarad, F.; Abdeljawad, T.; Baleanu, D. On the generalized fractional derivatives and their Caputo modification. J. Nonlinear Sci. Appl. 2017, 10, 2607-2619. [CrossRef]

25. Krasnoselskii, M.A. Two remarks on the method of successive approximations. Uspekhi Matematicheskikh Nauk 1955, 10, 123-127.

(C) 2019 by the authors. Licensee MDPI, Basel, Switzerland. This article is an open access article distributed under the terms and conditions of the Creative Commons Attribution (CC BY) license (http:// creativecommons.org/licenses/by/4.0/). 